PRAMANA

- journal of

physics
(C) Indian Academy of Sciences

Vol. 55, Nos $1 \& 2$

July \& August 2000

pp. $253-258$

\title{
Infra-red fixed points in supersymmetry
}

\author{
B ANANTHANARAYAN \\ Centre for Theoretical Studies, Indian Institute of Science, Bangalore 560 012, India \\ Email: anant@cts.iisc.ernet.in
}

\begin{abstract}
Model independent constraints on supersymmetric models emerge when certain couplings are drawn towards their infra-red (quasi) fixed points in the course of their renormalization group evolution. The general principles are first reviewed and the conclusions for some recent studies of theories with $R$-parity and baryon and lepton number violations are summarized.
\end{abstract}

Keywords. Supersymmetry; renormalization group; fixed points.

PACS Nos 11.10.Hi; 11.30.Fs; 12.60.Jv

\section{Introduction}

The Standard Model (SM) is a great success in describing the strong and electroweak interactions based on an underlying gauge principle, but one of its main weaknesses is that the masses of the matter particles, the quarks and leptons, are free parameters of the theory. This weakness persists in the minimal supersymmetric standard model (MSSM). The fermions mass problem in the MSSM arises from the presence of many unknown dimensionless Yukawa couplings. On the other hand the MSSM leads to a successful prediction for the ratio of the gauge couplings with a gauge unificiation scale $M_{G} \simeq 10^{16} \mathrm{GeV}$. It, therefore, becomes important to perform the radiative corrections in determining all the dimension $\leq 4$ terms in the lagrangian. This can be achieved by using the renormalization group equations in finding the values of parameters at the low scale, given their value at a high scale. Thus, considerable attention has recently been focussed on the renormalization group evolution [1] of the various dimensionless Yukawa couplings in the SM. Using the renormalization group evolution, one may attempt to relate the Yukawa couplings to the gauge couplings via the Pendleton-Ross infra-red stable fixed point (IRSFP) for the top-quark Yukawa coupling [2], or via the quasi-fixed point behaviour [3]. The predictive power of the SM and its supersymmetric extensions may, thus, be enhanced if the renormalization group (RG) running of the parameters is dominated by infra-red stable fixed points. Typically, these fixed points are for ratios like Yukawa couplings to the gauge coupling, or in the context of supersymmetric models, the supersymmetry breaking trilinear $A$-parameter to the gaugino mass, etc. These ratios do not attain their fixed point values at the weak scale, the range between the GUT (or Planck) scale and the weak scale being too small for the ratios to closely approach the fixed point. Nevertheless, the couplings may be determined by quasi-fixed point behaviour [3] where the value of the Yukawa coupling at 
the weak scale is independent of its value at the GUT scale, provided the Yukawa coupling at the GUT scale is large. For the fixed point or the quasi-fixed point scenarios to be successful, it is necessary that these fixed points are stable [4-6]. In the next section the above are reviewed and in the last the results pertaining to a theory with $R$-parity and baryon and lepton number violation are briefly summarized.

\section{Fixed points and stability}

In the SM, the top-quark Yukawa coupling $h_{t}$, satisfies the following one-loop evolution equation:

$$
8 \pi^{2} \frac{\mathrm{d} h_{t}}{\mathrm{~d} t}=h_{t}\left(9 g_{3}^{2}+\frac{3}{4}\left(3 g_{2}^{2}+g_{1}^{2}\right)+\frac{2}{3} g_{1}^{2}-\frac{9}{2} h_{t}^{2}\right),
$$

where $t \equiv \ln \left(\mu_{0}^{2} / \mu^{2}\right), \mu_{0}$ and $\mu$ are the initial and final momentum scales for the evolution and $g_{3}, g_{2}, g_{1}$ are the gauge couplings corresponding to $\mathrm{SU}(3), \mathrm{SU}(2), \mathrm{U}(1)$ respectively. Ignoring all except the strong gauge coupling constant $g_{3}$ allows us to write down the following equation for the ratio of the Yukawa and gauge coupling:

$$
8 \pi^{2} \frac{\mathrm{d} \ln \left(h_{t} / g_{3}\right)}{\mathrm{d} t}=g_{3}^{2}-\frac{9}{2} h_{t}^{2} .
$$

Setting the r.h.s. to zero yields the true fixed point for the top-quark Yukawa coupling in the SM, $h_{t}^{2}=2 g_{3}^{2} / 9$. We note here that at one-loop order, it is possible to discuss the solutions of the evolution equations even if the electro-weak gauge couplings are retained. In general we recall the following definitions:

$$
\frac{\mathrm{d} g_{i}^{2}}{\mathrm{~d} t}=-\frac{b_{i}}{16 \pi^{2}} b_{i} g_{i}^{4}, \frac{\mathrm{d} Y_{t}}{\mathrm{~d} t}=Y_{t}\left(\sum r_{i} \tilde{\alpha}_{i}-s Y_{t}\right), i=1,2,3,
$$

where $\tilde{\alpha}_{i}=g_{i}^{2} /\left(16 \pi^{2}\right), i=1,2,3 Y_{t}=h_{t}^{2} /\left(16 \pi^{2}\right)$. It may be shown that since the range of evolution of the couplings is finite the true fixed points are not reached in the infra-red regime, and instead quasi-fixed solution emerges. Furthermore, there is relative insensitivity to the initial values of the Yukawa couplings, the focussing property of the evolutions equations (this will be suitably illustrated in the next section). Retaining only the strong gauge coupling yields the following result for the quasi-fixed point at the scale $t$ :

$$
\left(\frac{Y_{t}}{\tilde{\alpha}_{3}}\right)^{\mathrm{QFP}}=\frac{\left(Y_{t} / \tilde{\alpha}_{3}\right)^{*}}{1-\left[\alpha_{3}(t) / \alpha_{3}(0)\right]^{B_{3}}}
$$

where $B_{3}=r_{3} / b_{3}+1$ and defines the rate of attraction, and $\left(Y_{t} / \tilde{\alpha}_{3}\right)^{*}=B_{3} b_{3} / s$ is the true fixed point value. In table 1 , we list the quantitites of interest for the $S M$ and the MSSM.

It turns out that in the MSSM the quasi-fixed point analysis yields a value for the topquark mass that is in surprisingly good agreement with the experimentally observed value and the rate of attraction to the true fixed point value is better. In practice, in the MSSM, 
Table 1.

\begin{tabular}{lcc}
\hline & SM & MSSM \\
\hline$b_{3}$ & -7 & -3 \\
$r_{3}$ & 8 & $16 / 3$ \\
$s$ & $9 / 2$ & 6 \\
$B_{3}$ & $-1 / 7$ & $-7 / 9$ \\
$\left(Y_{t} / \tilde{\alpha}_{3}\right)^{*}$ & $2 / 9$ & $7 / 18$ \\
\hline
\end{tabular}

the presence of the parameter $\tan \beta$ which can be $\gg 1$ and can yield phenomenologically desirable results [7], naturally requires us to consider a scenario where more than one Yukawa coupling can simultaneously reach fixed points. This would quantitatively alter the predictions for the top-quark mass slightly, and raises the important question of the stability of the fixed points of interest. The analysis rests on the stability of coupled first order differential equations and a thorough analysis can now be performed. In what follows, we shall only retain the strong gauge coupling and consider the system of evolution equations for the ratios $R_{i}=Y_{i} / \tilde{\alpha}_{3}$, with $i$ running over all the Yukawa couplings which satisfy the following evolution equations in a self-explanatory notation:

$$
\frac{\mathrm{d} R_{i}}{\mathrm{~d} t}=\tilde{\alpha}_{3} R_{i}\left[\left(r_{i}+b_{3}\right)-\sum S_{i j} R_{j}\right]
$$

where $r_{i}=\sum_{R} 2 C_{R}$, and $C_{R}$ stands for the color quadratic Casimir of the field. Fixed points arise when $R_{i}^{*}=0$ or when $R_{i}^{*}=\sum\left(S^{-1}\right)_{i j}\left(r_{j}+b_{3}\right)$. The stability of the solutions may be tested by linearizing the system about the fixed points. For the non-trivial fixed points we need to consider the eigenvalues of the stability matrix whose elements are given by $A_{i j}=R_{i}^{*} S_{i j} / b_{3}$. The fixed point is infra-red stable if the real part of each of the eigenvalues is negative. For the trivial fixed points the linear stability analysis requires that each $\lambda_{i}=\left(\sum_{j} S_{i j} R_{j}^{*}-\left(r_{i}+b_{3}\right)\right) / b_{3}$ also be negative. In practice, it turns out that all Yukawa couplings that are associated with colored fields are required to attain non-trivial fixed points, and those Yukawa couplings which are associated with colored singlets attain trivial fixed points in order for the fixed points to be infra-red stable, at least in models of interest. The extension of the analysis above to the soft supersymmetry breaking sector may also be done. In particular, for the ratio of the tri-linear couplings to the gaugino mass, the analysis is particulary straightforward. A close scrutiny of the evolution equations for these reveals that in the event that some of the Yukawa couplings attain non-trivial fixed points, the ratio $A_{i} / m_{\tilde{g}}$ attains the value of 1 , and values related to the values of fixed point Yukawa couplings for all those that correspond to trivial fixed points. Moreover, the stability analysis need not be carried afresh and corresponds to that of the Yukawa coupling sector.

\section{Models with $\boldsymbol{R}$-parity and baryon and lepton number violation}

Here we will summarize briefly the results of some recent investigations involving $R$-parity and baryon and lepton number violation. We recall that $R$-parity is a discrete symmetry imposed on the MSSM to prevent rapid proton decay through dimension 4 operators [8]. One may add all terms consistent with gauge symmetry and supersymmetry and introduce the following lepton and baryon number violating terms to the superpotential: 


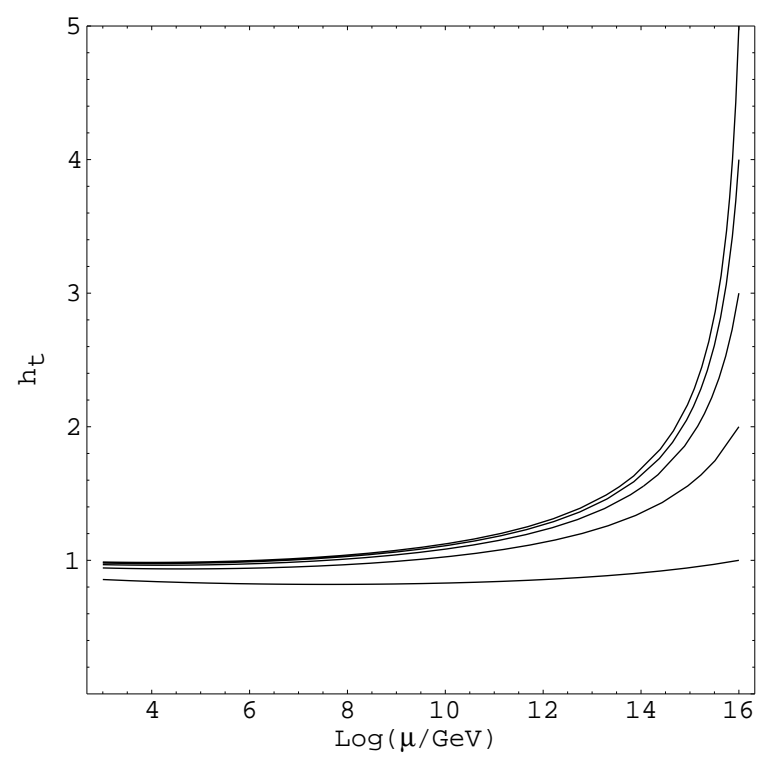

Figure 1. Renormalization group evolution of the top-quark Yukawa coupling $h_{t}$ as a function of the logarithm of the energy scale. We have taken the initial values of $h_{t}$ at the scale $M_{G} \sim 10^{16}$ to be 5.0,4.0, 3.0, 2.0, and 1.0. The initial values of other Yukawa couplings are $h_{b}=0.91, h_{\tau}=0$, and $\lambda_{233}^{\prime \prime}=1.08$.

$$
\begin{aligned}
& W_{L}=\frac{1}{2} \lambda_{a b c} L_{L}^{a} L_{L}^{b} \bar{E}_{R}^{c}+\lambda_{a b c}^{\prime} L_{L}^{a} Q_{L}^{b} \bar{D}_{R}^{c}+\mu_{i} L_{i} H_{2}, \\
& W_{B}=\frac{1}{2} \lambda_{a b c}^{\prime \prime} \bar{D}_{R}^{a} \bar{D}_{R}^{b} \bar{U}_{R}^{c},
\end{aligned}
$$

respectively, where $L, Q, \bar{E}, \bar{D}, \bar{U}$ denote the lepton and quark doublets, and anti-lepon singlet, $d$-type anti-quark singlet and $u$-type anti-quark singlet, respectively. Statistics and proton decay constraints will restrict us to consider the following couplings $\lambda_{233}, \lambda_{333}^{\prime}, \lambda_{233}^{\prime \prime}$ only. The fixed point behaviour of these couplings has been considered in great detail in the recent past $[9,10]$. It turns out that there is only a class of solutions that gives physically acceptable results and is infra-red stable and corresponds to one where, $R_{\tau}^{*}=0, R^{\prime \prime *}=77 / 102, R_{t}^{*}=R_{b}^{*}=2 / 17$. (The theory may be enhanced to include one of the $L$ violating coupling $R^{*}=0$ which remains indistinguishable from the first case.) It may also be seen in a straightforward manner that the ratio of the tri-linear couplings to the gluino masses attain their fixed point values: $\tilde{A}_{\lambda^{\prime \prime}}^{*}=\tilde{A}_{b}^{*}=\tilde{A}_{t}^{*}=1$, and $\tilde{A}_{\tau}^{*}=-2 / 17$, where the latter is intimately related to the values of the fixed points $R_{b}^{*}$.

We finally illustrate several of the remarks made during the course of this discussion in figures 1 and 2. The focussing property of the one-loop evolution equations for the Yukawa couplings is brought out in figure 1, where the running Yukawa coupling is plotted as a function of the logarithm of the momentum scale. We see that for a range of values of the Yukawa coupling at the unification scale, the evolution equations focus them to the 


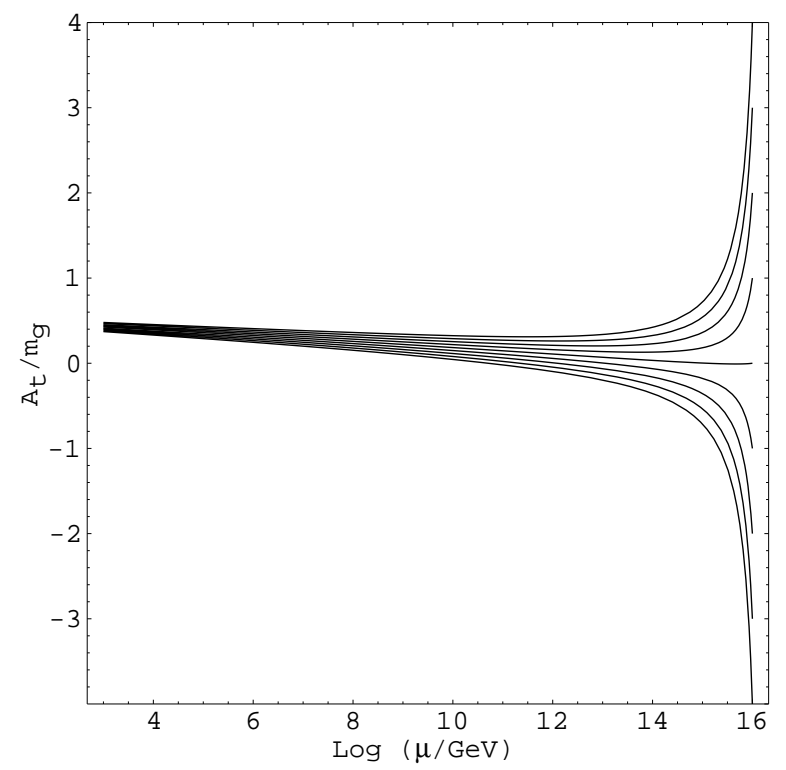

Figure 2. Renormalization group evolution of ratio $A_{t} / m_{\tilde{g}}$, as a function of the $\log$ arithm of the energy scale for several different initial values at $M_{G}$. The initial values for other parameters at $M_{G}$ are $h_{t}=5.0, h_{b}=0.91, h_{\tau}=0, \lambda_{233}^{\prime \prime}=1.08$, and $A_{b} / m_{g}=1.94, A_{\lambda^{\prime \prime}} / m_{g}=2.57$.

neighbourhood of the quasi-fixed point. This is also true for the $b$-quark and baryon number violating coupling Yukawa couplings $h_{b}, \lambda_{233}^{\prime \prime}$ which are not shown here (a complete discussion can be found in ref. [10]). We illustrate the behaviour of the ratio $\tilde{A}_{t}$ plotted as a function of the logarithm of the momentum scale. The focussing property is also well illustrated in this case. In conclusion, we note that the presence of infra-red stable fixed points in supersymmetric models provides a valuable guide to the possible range of parameters of the model which are model independent. In the MSSM the top-quark mass comes out in the range predicted by these considerations. $R$-parity and baryon and lepton number violating models and the range of parameters therein, can be constrained to a narrow class that is favored by infra-red stable fixed points.

\section{References}

[1] For a review and references, see e.g., B Schrempp and M Wimmer, Prog. Part. Nucl. Phys. 37, 1 (1996)

[2] B Pendleton and G G Ross, Phys. Lett. B98, 291 (1981) M Lanzagorta and G G Ross, Phys. Lett. B349, 319 (1995)

[3] C T Hill, Phys. Rev. D24, 691 (1981)

[4] B C Allanach and S F King, Phys. Lett. B407, 124 (1997)

[5] S A Abel and B C Allanach, Phys. Lett. B415, 371 (1997)

[6] I Jack and D R T Jones, Phys. Lett. B443, 177 (1998) 


\section{B Ananthanarayan}

[7] B Ananthanarayan, G Lazarides and Q Shafi, Phys. Rev. D44, 1613 (1991)

[8] S Weinberg, Phys. Rev. D26, 287 (1982)

N Sakai and T Yanagida, Nucl. Phys. B197, 133 (1982)

[9] B Ananthanarayan and P N Pandita, Phys. Lett. B454, 84 (1999)

[10] B Ananthanarayan and P N Pandita, hep-ph/9911464 